PROGRESS IN BIOPHYSICS AND MOLECULAR BIOLOGY

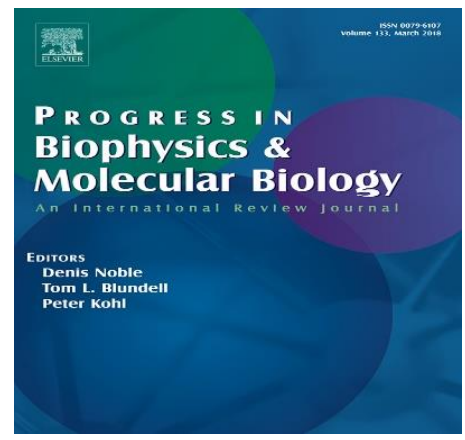

Editor-In-Chief:

Prof. Tom Blundell, Dept. Biochemistry, University of Cambridge, UK.

ISSN: 0079-6107; Impact Factor: 3.227; Publisher: Elsevier UK

Accepted-March $8^{\text {th }} 2018$

\title{
PHYSICS OF FRACTIONAL IMAGING IN BIOMEDICINE
}

\author{
Ayesha Sohail $^{\mathrm{a} *}$, O. Anwar Bég ${ }^{\mathrm{b}}$, Zhiwu Li ${ }^{\mathrm{c}, \mathrm{d}}$ and Khadija Maqbool ${ }^{\mathrm{e}}$ \\ ${ }^{a}$ Department of Mathematics, Comsats Institute of Information Technology, Lahore 54000, Pakistan \\ ${ }^{b}$ Fluid Mechanics, Bio-Propulsion and Nanosystems, Aeronautical and Mechanical Engineering Department, University \\ of Salford, Newton Building, UG17, Manchester, M54WT, UK. (e-mail: O.A.Beg@salford.ac.uk) \\ 'School of Electro-Mechanical Engineering, Xidian University, Xi'an 710071, China \\ ${ }^{d}$ Institute of Systems Engineering, Macau University of Science and Technology, Taipa, Macau \\ ${ }^{e}$ Department of Mathematics \& Statistics, International Islamic University, Islamabad 44000, Pakistan
}

*Corresponding author. E-mail address: asohail@ciitlahore.edu.pk

\begin{abstract}
Medical imaging is a rapidly evolving sub-field of biomedical engineering as it considers novel approaches to visualizing biological tissues with the general goal of improving health. Medical imaging research provides improved diagnostic tools in clinical settings and thereby assists in the development of drugs and other therapies. Data acquisition and diagnostic interpretation with minimum error are important technical aspects of medical imaging. The image quality and resolution are critical in visualization of the internal aspects of patient's body. Although a number of user-friendly resources are available for processing image features, such as enhancement, colour manipulation and compression, the development and refinement of new processing methods is still a worthwhile endeavour. In this article we aim to highlight the role of fractional calculus in imaging with the aid of a variety of practical examples.
\end{abstract}

KEY WORDS: Biomedical engineering; diagnostic tools; fractional calculus in imaging; DW-MRI; tissue; brain scans. 


\section{INTRODUCTION TO IMAGING IN MEDICINE}

During the past two decades, the "imaging" subfield of biomedical engineering has received a considerable attention, not only from biomedical engineers but also from medical physicists and mathematicians. This response has served to improve the techniques utilized and additionally has expanded this field of research into new frontiers. With the passage of time, advanced imaging techniques are continuously being introduced into the field of bioengineering with enhanced cost effectiveness and non-invasive properties. Several steps such as biomaterial development, analytical methodologies and research on the application of concepts (interfacing engineering, computing, mathematics, chemical and physical sciences) are intrinsic to the development and success of novel ideas of biomedical engineering. Thus due to the successful interface between different disciplines, robust tools for diagnosis and therapy for human diseases have developed tremendously. To obtain images of internal aspects of the body, the basic imaging techniques which have been developing since 1895 include radiology, ultrasonography and magnetic resonance. To overcome the side effects of such techniques, different measures have been made and new advanced techniques have been introduced. These include ultrasonography, which is the cheapest and most harmless technology in radiology. Other methods include magnetic resonance imaging (MRI) which makes use of the potential energy stored in the body's hydrogen atoms, such atoms are manipulated by very strong magnetic fields and radiofrequency pulses to produce adequate amount of localizing and tissue-specific energy that will be used by highly sophisticated computer programs in order to generate 2D and 3D images. Further techniques include Nuclear medicine images (NMI) which are generated by giving the patient a short-lived radioactive material, and then using $\gamma$-camera or a positron emission scanner that records radiation emanating from the patient and finally the PET/CT technique which comprises the integration of PET functional nuclear medicine data with $\mathrm{CT}$ anatomic data (PET/CT). All the techniques 
of medical imaging depend on different factors, of which probably the most crucial are: the technical aspects of data acquisition and diagnostic interpretation with minimum error. The image quality and resolution is extremely important since it permits an accurate rendering of the patient's internal body aspects. Although there are several user-friendly resources for processing image features, such as enhancement, colour manipulation and compression, the development of new processing methods still warrants the attention of researchers. Different mathematical models have been used in this field of research, both in the classical integer order and in the fractional order sense. Fractional calculus is approximately a three century old field of study and is a branch of mathematics that deals with non-integer order differential and integral operators. The last few decades have seen a massive surge in interest geared towards applications of fractional calculus in many fields of science, engineering and biotechnology [1, 2, 3]. Fractional order mathematical models have been used in the field of biological sciences to understand many complex phenomena. There have been different types of definitions of fractional derivatives, and different fractional derivatives are usually associated with different discretization schemes and possess different stability and convergence criteria [4]. The most commonly used definitions are the Riemann-Liouville and Caputo fractional derivative. In view of the fact that the initial-value condition for the Riemann-Liouville definition can be written in a fractional derivative form, the physical meaning is not articulate. On the other hand the condition for Caputo version is given in classical integer-order derivative form and has an explicit physical meaning. Consequently, the fractional derivative $D_{t}^{\alpha}$ in the Caputo approach is frequently used in the mathematical biology literature [5]. In this article we aim to present the role of fractional calculus in imaging with the aid of practical examples such as fractional differential calculus for enhancing and restoring image quality [6], fractional order viscoelasticity models for elastography imaging [7], fractional order differential operators 
expressing anomalous diffusion [8,9], diffusion weighted imaging for tissues [10] and other applications.

\section{MEDICAL IMAGING AND ADAPTIVE FRACTIONAL DIFFERENTIATION}

It has been reported that, compared with traditional integral differential approaches, "fractional differentials" when applied to image processing can enhance edges, make texture details clearer, and preserve smooth areas. Therefore, medical images processed by fractional differentials are sharper and have higher contrast. For example, work conducted by $\mathrm{Pu}$ et. al [11], demonstrates the importance of fractional calculus in imaging with the aid of masks (figure 1). For the edge processing, the traditional fractional differentials use the same fractional order, textures and smooth areas of image. When edges would be enhanced by high fractional orders, weak textures and smooth areas would be ignored. Alternatively, weaker textures and smoother areas would be preserved by low fractional orders, such that edges would be weakened. Therefore image enhancement is a challenging task. To address such difficulties, traditional and improved fractional differential algorithms have been developed for digital image processing [12]. Similarly adaptive fractional derivatives have also been used for the image de-noising cases [13]. The methods of image enhancement via fractional calculus can prove to be helpful in the field of medicine and biology, by improving the image quality. The recently proposed adaptive fractional differential approach (AFDA) [6] considers global and local information in medical images and yields better enhancement effects than traditional image processing methods. The algorithm adapted in their formulation works by segmenting every area of the image accurately and processes every pixel of an image (based on the characteristics of local information specific to that pixel). This technique focuses on both the fractional derivatives and the area based image features. A large number of simulation experiments have shown that AFDA achieves the best enhancement effect of medical images for medical diagnoses. Therefore the fractional calculus can help to resolve medical imaging 
issues, such as low contrast and significant noise. In contrast to the fractional differentials used in this study, fractional integrals can also prove to be helpful in attenuating high frequency components of a signal and in enhancing low frequency components; therefore, fractional integrals can remove image noise while preserving texture information. The adaptive fractional integral algorithm for image de-noising is an open problem, and when designed in conjunction with the developed adaptive fractional differential algorithm (to enhance and preserve image texture) can prove to be more fruitful. In figure 2, the comparative study of adaptive results for an orthopaedic application i.e. the knee joint is presented [6].

\section{TISSUE VISCOELASTICITY AND FRACTIONAL ORDER IMAGING}

A recently described approach for elasticity imaging, using propagating acoustic shear waves and phase-contrast MRI, has been called magnetic resonance elastography (MRE). Magnetic Resonance Elastography (MRE) is an imaging technique that captures the viscoelastic properties of soft biological tissues in complex organs such as the brain and liver. An important step in this analysis is the reconstruction of material properties through dynamic displacement imaging. For the execution of such reconstruction algorithms in these techniques, certain viscoelastic material models are used. A model that demonstrates accurately the viscoelasticity of soft tissue-like materials could improve the diagnostic capability of elastography. Researchers have explored this field initially through classical integer order models and more recently through fractional order derivatives. These models include the classical dashpot viscoelastic models of Voigt and Kelvin [14], more complex rheological models [15] and standard linear solid models [15] for the non-invasive measurement of viscoelasticity of different organs including the brain (cerebellum), liver, and as a diagnostic technique for detecting cancer and other diseases. The fractional order models have also been used to study MRE. In this regard Meral et. al. [7] approximated the viscoelastic material behavior of materials through the corresponding fractional differential equations and compared fractional 
and integer order models to describe their behavior under harmonic mechanical loading. During MRE, external force (or pressure) is applied to image the deformation and to identify structural mechanical properties. This makes the modelling of elastic, viscous and inertial properties very important. Studies have shown that the integer derivative viscoelastic models (such as Kelvin and Voigt) based on a combination of purely elastic and viscous elements, have limitations, particularly for biological tissues and tissue mimicking phantoms when a range of frequencies of motion are considered, and that fractional order viscoelasticity models with fewer parameters may be more efficient in describing rheological tissues.

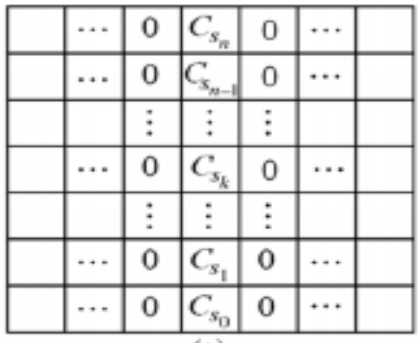

(a)

\begin{tabular}{|c|c|c|c|c|c|c|}
\hline & $\cdots$ & 0 & $C_{s_{0}}$ & 0 & $\cdots$ & \\
\hline & $\cdots$ & 0 & $C_{s_{1}}$ & 0 & $\cdots$ & \\
\hline & & $\vdots$ & $\vdots$ & $\vdots$ & & \\
\hline & $\cdots$ & 0 & $C_{s_{k}}$ & 0 & $\cdots$ & \\
\hline & & $\vdots$ & $\vdots$ & $\vdots$ & & \\
\hline & $\cdots$ & 0 & $C_{s_{n-1}}$ & 0 & $\cdots$ & \\
\hline & $\cdots$ & 0 & $C_{s_{n}}$ & 0 & $\cdots$ & \\
\hline
\end{tabular}

(c)

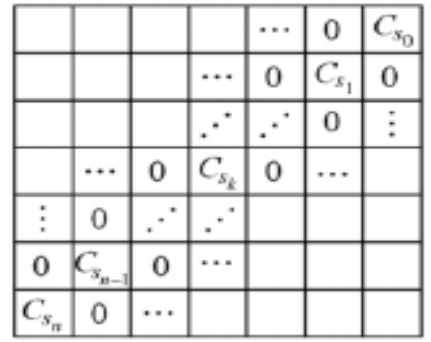

(e)

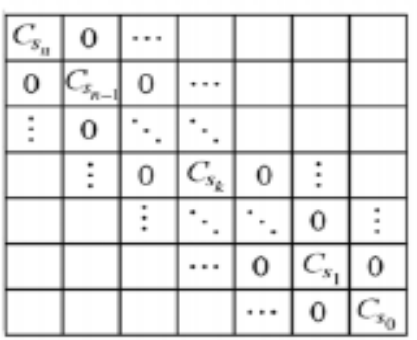

(g)

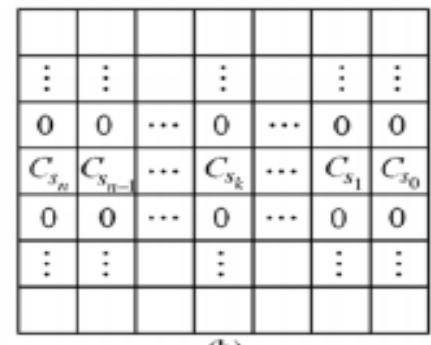

(b)

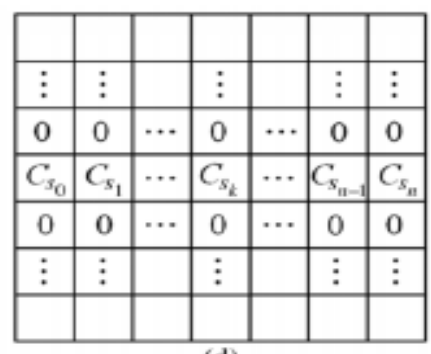

(d)

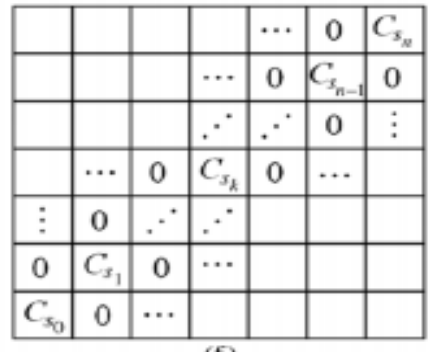

(f)

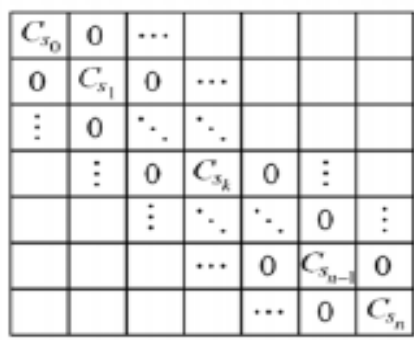

(h) 
Figure 1: To obtain the fractional differential on the eight symmetric directions and make the fractional differential masks have anti-rotation capability, 8 fractional differential masks which are respectively on the directions of negative $\mathrm{x}$-coordinate, negative $\mathrm{y}$-coordinate, positive $\mathrm{x}$ coordinate, positive y-coordinate, left downward diagonal, right upward diagonal, left upward diagonal, and right downward diagonal are implemented, Csn is the mask coefficient on interest pixel [11] (with permission).

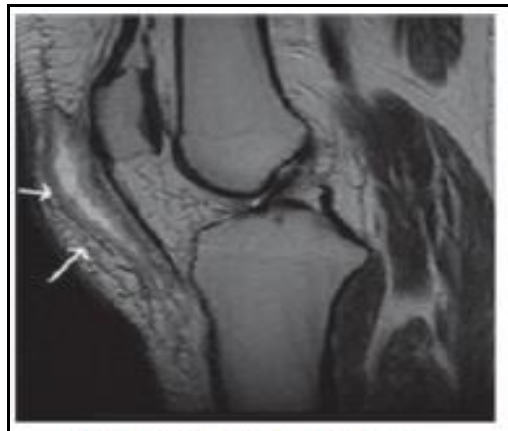

(a) Original image

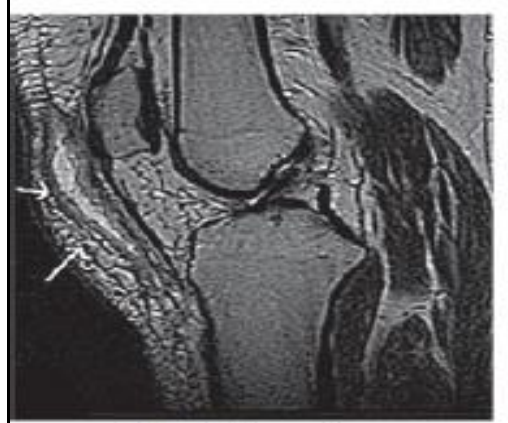

(d) 0.8 -order

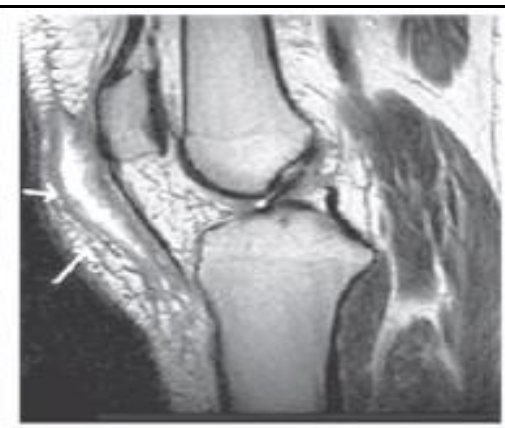

(b) Histogram

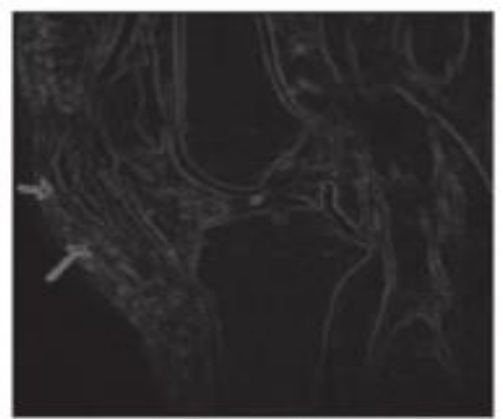

(e) 1-order

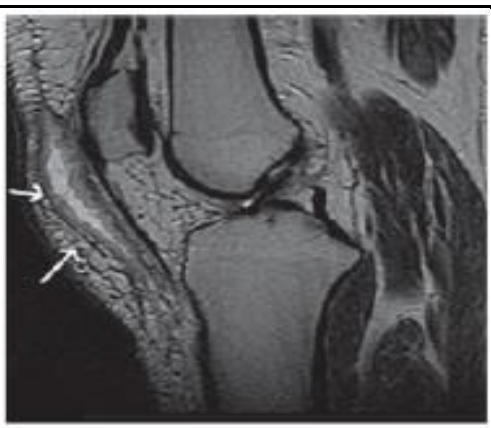

(c) 0.5 -order

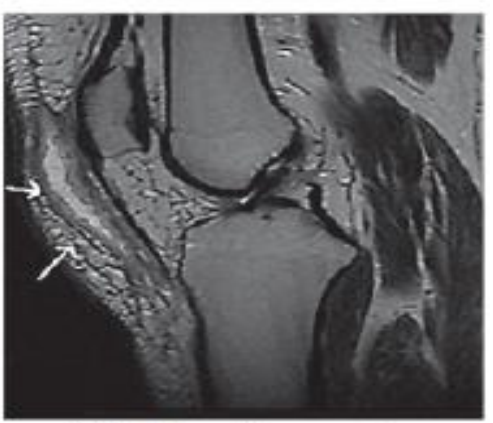

(f) Adaptive-order

Figure 2: Magnetic image resonance enhancement via adaptive fractional modelling [6].

Craiem \& Armentano [16] proposed two fractional alternative models with one and two springpots. The models were tested in a control state and during smooth muscle activation in animal experiments. Their results confirmed the experimental findings to be consistent with fractional model predictions. Subsequently after several other studies adapted the fractional approach, Xiao [17] examined the effect of varying fractional order parameter (of viscoelasticity) on the dependence of afP $W V$ on HR. 
Diffusion-weighted magnetic resonance imaging (DWI or DW-MRI) is an imaging method that uses the diffusion of water molecules to generate contrast in MR images. It allows the mapping of the diffusion process of molecules, mainly water, in biological tissues, in vivo and non-invasively. It has been reported [18] that this technique has the capability of detecting early or subtle changes within the brain before any visible abnormality can be seen on conventional morphologic imaging. There is growing interest in the application of DWI for the evaluation of the patient with cancer. Due to its fast application (typically several minutes) and independence from administration of exogenous contrast medium and quantitative properties, this methodology can be greatly assistive in tumour assessment. The software tools that are available for quantitative analysis on most commercial platforms can be improved, to deal with complex processes, with the help of fractional anisotropy, perfusion fraction, different noise filtration techniques and image registration techniques. Recent research has confirmed that DWI has the ability to detect inherent tissue contrast for the oncologic problems. Following its success in the field of neuro-oncology, DWI is achieving substantial success in oncologic imaging of the prostate gland, breast and liver. A detailed explanation of the biophysical basis of diffusion contrast, the difference between hindered and restricted diffusion, and the derivation of diffusion parameters is documented in White et. al. [19], wherein an advanced DWI modeling technique, i.e. restriction spectrum imaging (RSI) has also been described. The Bloch equation is a phenomenological description of the precessional motion and relaxation of the magnetization arising from nuclear magnetic moment spins. Solving the Bloch equation for different combinations of static, radiofrequency and gradient magnetic fields provides the basis for NMR spectroscopy and MRI. The correlation between developing pathology and localized diffusion relies on diffusion-weighted pulse sequences to probe biophysical models of molecular diffusion, which is typically defined as an exponential function of apparent diffusion coefficient $A$ and specific gradient pulse sequence parameter $p$ : 
$D_{m}=e^{-A P}$

and the stretched anomalous diffusion as:

$D_{m}=e^{-(A P) \alpha}$

where $\alpha$ is a measure of tissue complexity that can be derived from fractal models of tissue structure. An alternative derivation of equation 2 is discussed in [20] where the spatial Laplacian in the Bloch-Torrey equation is generalized to incorporate a fractional order Brownian model of diffusivity. The case where the time derivative in the Bloch Torrey equation is replaced by a "Riemann-Liouville" fractional order time derivative expressed in the Caputo form" has also been discussed.

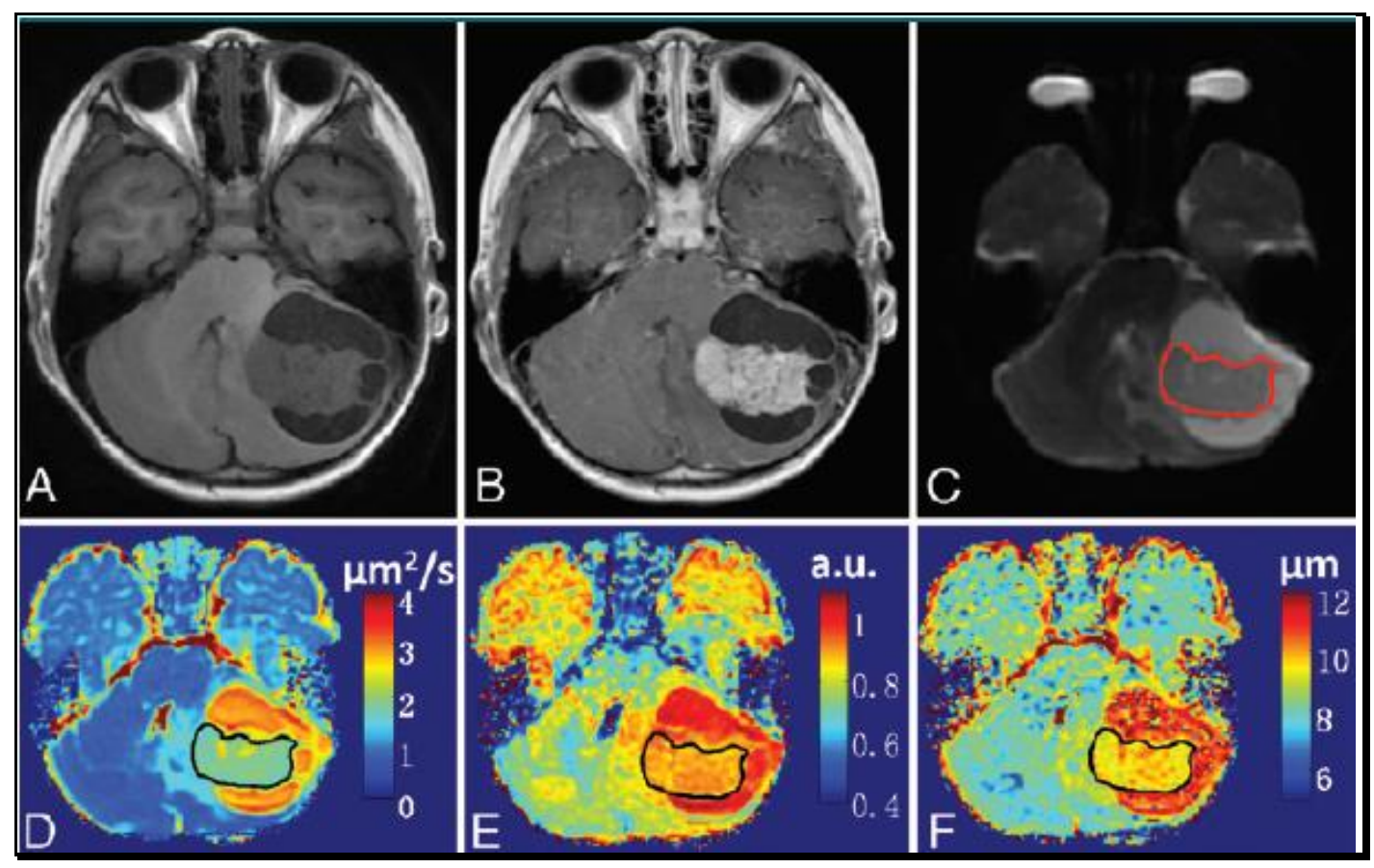

Figure 3: Low-grade brain tumour (WHO grade II astrocytoma) in the left cerebellum of a 4year-old boy. Top row: A, axial unenhanced T1-weighted FLAIR; B, contrast-enhanced T1weighted FLAIR; and, C, T2-weighted echoplanar MR images show an enhancing lesion surrounded by cysts. Bottom row: MR images with quantitative FROC maps show, D, D values; E, b values; and, F, m values. The solid tumour is enclosed within the ROI (red area in 
C ), which was initially drawn on the T2-weighted echoplanar image with the aid of T1weighted images and propagated into the FROC maps. The D, b, and $m$ values within the ROI are higher than those for a high-grade brain tumour [21] (with permissions)

The results showed that both cases revert to the classical results for integer order operations. Fractional order dynamics derived for the first case were observed to fit the signal attenuation in DWI obtained from Sephadex gels, human articular cartilage and human brain. Future developments of this approach may be useful for classifying anomalous diffusion in tissues with developing pathology. Recently Karaman and his collaborators [22] have demonstrated that the fractional motion diffusion model (FM diffusion model) is reliable over the conventional ADC approach, when used with high parametric values to improve differentiating between low and high grade paediatric brain tumours. They reported that FM diffusion model offers higher specificity, sensitivity, and diagnostic accuracy. Cancer is a growing threat and imaging is helping to identify the invasion and metastasis thresholds of this deadly disease, specifically for the soft tissues. Differentiating low from high-grade brain tumours without the use of invasive biopsy is important for optimizing patient management strategies and determining the time point when benign tumors begin to transform into malignant lesions so that timely interventions can be applied without unnecessarily compromising patient quality of life. The correct time management for the treatment of the tumour, at correct and initial growth stages may save patient's life. The identification of the critical stage, when the tumour transforms to malignant from benign, has remained a challenge for the oncologists. Recently, a leading research group led by Sui [21] demonstrated with the aid of new parameters of fractional calculus, the improved accuracy which is attainable while studying such stages. They proposed that with the help of fractional order calculus (FROC) diffusion model, the accuracy of MR imaging can be improved, when used to differentiate among low- and high-grade paediatric brain tumours. The objective in deploying a fractional order calculus (FROC) diffusion model was to overcome the shortcomings of the other available methods such as the 
mono-exponential diffusion model [23], which have been used for the high-grade tumours. To achieve the limiting values for the apparent diffusion coefficient (ADC) and to describe the anomalous diffusion process accurately tissues, Sui et. al. [21] presented a detailed analysis.

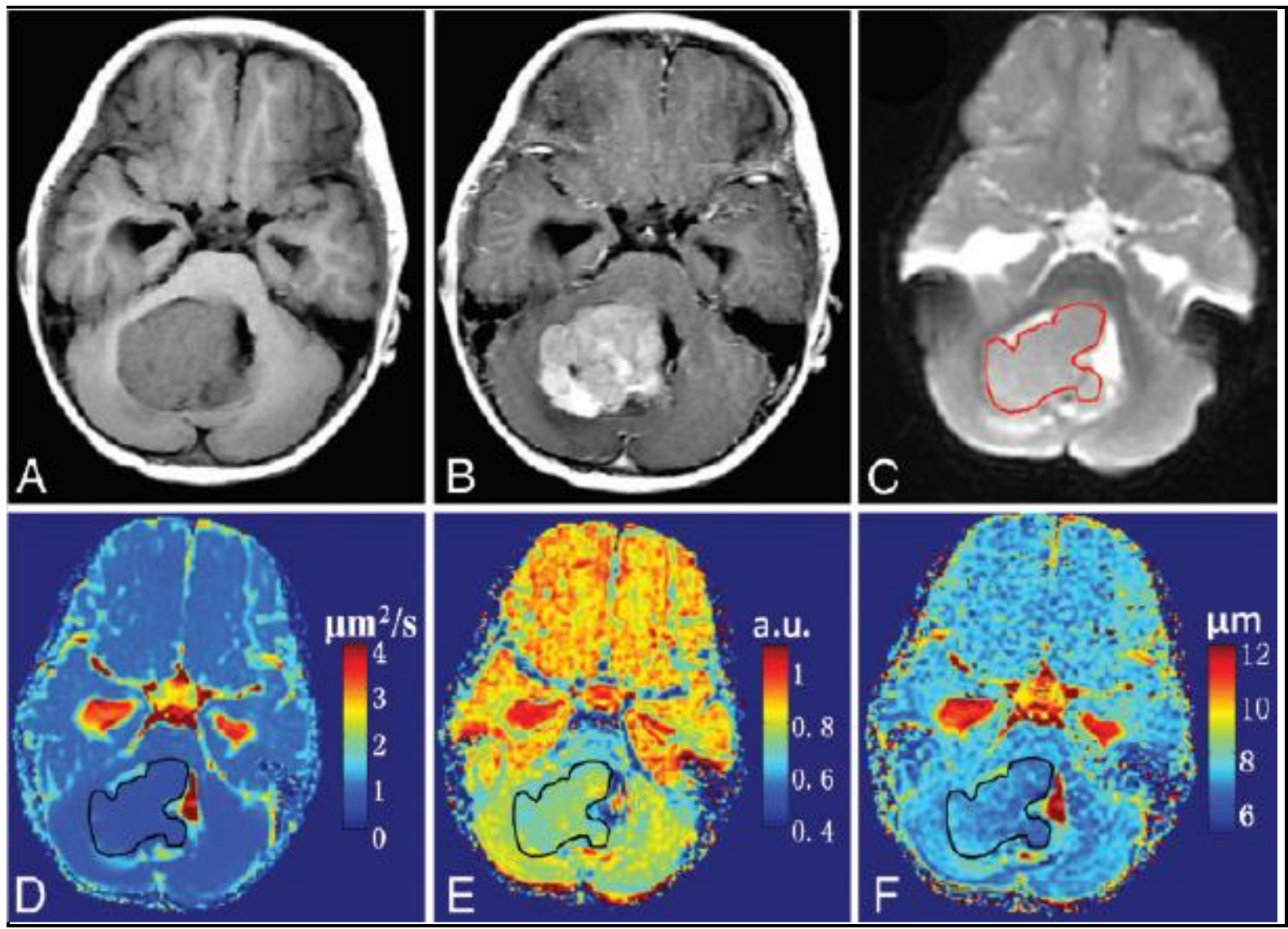

Figure 4: High-grade brain tumour (WHO grade IV medulloblastoma) in the right cerebellum of a 6-year -old boy. Top row: A, axial unenhanced T1-weighted FLAIR; B, contrast-enhanced T1-weighted FLAIR; and, C, T2-weighted echoplanar MR images show an enhancing lesion. Bottom row: MR images with quantitative FROC maps show, D, D values; E, b values; and, $\mathrm{F}, \mathrm{m}$ values. The solid tumor is enclosed with the ROI (red area in C), which was initially drawn on the T2-weighted echoplanar image with the aid of T1-weighted images and propagated into the FROC maps. The signal characteristics of anatomic images (A-C) are similar to those of the low-grade tumour (cf Fig 1). However, the FROC maps ( D-F) show substantial differences between low- and high-grade tumours [21] (with permissions).

The fractional technique they adopted was linked with a set of parameters including the diffusion coefficient, the space fractional order derivative and an additional spatial derivative 
(to demonstrate the diffusion as well as the porous structure of the tissue through which the water molecules diffuse). Some results from this study are presented in figures 3 (for low grade brain tumour) and 4 (for low grade), where the fractional order calculus (FROC) diffusion model reveals some more realistic results. Several other models and methods are under development in the continuation of the available methods. For example the fractional lattice Boltzmann method has been introduced recently [24]. On the other hand, the Lattice Boltzmann method (LBM) has been used for the two-step computational analysis of magnetic resonance flow imaging [25]. The first model component provides fluid dynamic maps by applying LBM. The second one couples MR imaging (MRI) modelling with a new magnetization transport algorithm based on the Eulerian coordinate approach and uses the flow maps. This technique was verified to be an efficient approach with fast computations and the results were shown to be in close agreement with the experimental findings. The MRI experiments were conducted by the spoiled gradient echo sequence (FLASH), with and without flow compensation along the slice-selective and frequency-encoding directions. It is anticipated that the fractional order LBM when applied to MRI can improve markedly the image properties.

\section{CONCLUSIONS}

In the field of imaging, mathematical models have always contributed positively and have worked as smart and efficient tools. Iirrespective of whether they are Poisson image analysis, gradient domain high dynamic range compression, colour to gray imaging or for random walks for interactive Alpha-Matting, the crucial role of mathematical tools in optimizing imaging processing cannot be denied. Particularly, with the advancement in the field of fractional imaging, several tools and libraries have been developed and are available online, to improve the image quality $[26,27]$. A selective and recent collection of fractional-order based image 
processing methods have been reviewed in this survey to emphasize the importance of the methodology of fractional order image processing and fractional order tuning. It is anticipated, that specifically the fractional order modelling will prove to be more beneficial in medicine and biology in particular in the area of non-invasive studies.

\section{REFERENCES}

[1] Y. Zhou, J. Wang, and L. Zhang, Basic Theory of Fractional Differential Equations. World Scientific, 2016.

[2] D. Baleanu, K. Diethelm, E. Scalas, and J. J. Trujillo, Fractional Calculus: Models and Numerical Methods, vol. 5. World Scientific, 2016.

[3] R. P. Agarwal, M. Benchohra, and S. Hamani, "A survey on existence results for boundary value problems of nonlinear fractional differential equations and inclusions," Acta Applicandae Mathematicae, vol. 109, no. 3, pp. 973-1033, 2010.

[4] A. McBride, J. Sabatier, O. Agrawal, and J. T. Machado (Editors), “Advances in fractional calculus: theoretical developments and applications in physics and engineering," Springer, Germany, 2008.

[5] "O. Gu"ner and A. Bekir, "Exact solutions of some fractional differential equations arising in mathematical biology," International Journal of Biomathematics, vol. 8, no. 01, p. 1550003, 2015.

[6] B. Li and W. Xie, “Adaptive fractional differential approach and its application to medical image enhancement," Computers \& Electrical Engineering, vol. 45, pp. 324- 335, 2015. 
[7] F. Meral, T. Royston, and R. Magin, "Fractional calculus in viscoelasticity: an experimental study," Communications in Nonlinear Science and Numerical Simulation, vol. 15, no. 4, pp. 939-945, 2010.

[8] R. Klages, G. Radons, and I. M. Sokolov, Anomalous Transport: Foundations and Applications, John Wiley \& Sons, New York, 2008.

[9] Y. Liang, Q. Y. Allen, W. Chen, R. G. Gatto, L. Colon-Perez, T. H. Mareci, and R. L. Magin, "A fractal derivative model for the characterization of anomalous diffusion in magnetic resonance imaging," Communications in Nonlinear Science and Numerical Simulation, vol. 39, pp. 529-537, 2016.

[10] C. Ingo, R. L. Magin, L. Colon-Perez, W. Triplett, and T. H. Mareci, “On random walks and entropy in diffusion-weighted magnetic resonance imaging studies of neural tissue," Magnetic Resonance in Medicine, vol. 71, no. 2, pp. 617-627, 2014.

[11] Y.-F. Pu, J.-L. Zhou, and X. Yuan, "Fractional differential mask: a fractional differentialbased approach for multiscale texture enhancement," IEEE Transactions on Image Processing, vol. 19, no. 2, pp. 491-511, 2010.

[12] B. Li and W. Xie, "Adaptive fractional differential algorithm based on otsu standard," in $26^{\text {th }}$ Control and Decision Conference (2014 CCDC), pp. 2020-2025, IEEE, 2014.

[13] R. Chan, A. Lanza, S. Morigi, and F. Sgallari, “An adaptive strategy for the restoration of textured images using fractional order regularization," Numerical Mathematics: Theory, Methods and Applications, vol. 6, no. 01, pp. 276-296, 2013.

[14] K. Marynowski and T. Kapitaniak, "Kelvin-Voigt versus Burgers internal damping in modeling of axially moving viscoelastic web," International Journal of Non-Linear Mechanics, vol. 37, no. 7, pp. 1147-1161, 2002. 
[15] P. Asbach, D. Klatt, U. Hamhaber, J. Braun, R. Somasundaram, B. Hamm, and I. Sack, "Assessment of liver viscoelasticity using multifrequency MR elastography," Magnetic Resonance in Medicine, vol. 60, no. 2, pp. 373-379, 2008.

[16] D. Craiem and R. L. Armentano, "A fractional derivative model to describe arterial viscoelasticity,” Biorheology, vol. 44, no. 4, pp. 251-263, 2007.

[17] H. Xiao, I. Tan, M. Butlin, D. Li, and A. P. Avolio, "Arterial viscoelasticity: role in the dependency of pulse wave velocity on heart rate in conduit arteries," American Journal of Physiology-Heart and Circulatory Physiology, vol. 312, no. 6, pp. H1185- H1194, 2017.

[18] S. Zhang, D. H. Laidlaw, and G. Kindlmann, "Diffusion tensor MRI visualization," The Visualization Handbook, pp. 327-340, 2004.

[19] N. S. White, C. R. McDonald, N. Farid, J. Kuperman, D. Karow, N. M. Schenker, Ahmed, H. Bartsch, R. Rakow-Penner, D. Holland, A. Shabaik, et al., "Diffusion weighted imaging in cancer: physical foundations and applications of restriction spectrum imaging," Cancer Research, vol. 74, no. 17, pp. 4638-4652, 2014.

[20] R. L. Magin, O. Abdullah, D. Baleanu, and X. J. Zhou, “Anomalous diffusion expressed through fractional order differential operators in the Bloch-Torrey equation," Journal of Magnetic Resonance, vol. 190, no. 2, pp. 255-270, 2008.

[21] Y. Sui, H. Wang, G. Liu, F. W. Damen, C. Wanamaker, Y. Li, and X. J. Zhou, "Differentiation of low-and high-grade paediatric brain tumours with high b-value diffusion weighted MR imaging and a fractional order calculus model," Radiology, vol. 277, no. 2, pp. 489-496, 2015. 
[22] M. M. Karaman, H. Wang, Y. Sui, H. H. Engelhard, Y. Li, and X. J. Zhou, “A fractional motion diffusion model for grading paediatric brain tumors," NeuroImage: Clinical, vol. 12, pp. 707-714, 2016.

[23] S. Riches, K. Hawtin, E. Charles-Edwards, and N. De Souza, "Diffusion-weighted imaging of the prostate and rectal wall: comparison of biexponential and monoexponential modelled diffusion and associated perfusion coefficients," NMR in Biomedicine, vol. 22, no. 3, pp. 318-325, 2009.

[24] J. Zhou, P. M. Haygarth, P. Withers, C. Macleod, P. D. Falloon, K. J. Beven, M. C. Ockenden, K. J. Forber, M. J. Hollaway, R. Evans, et al., "Lattice Boltzmann method for the fractional advection-diffusion equation," Physical Review E, vol. 93, no. 4, p. 043310, 2016.

[25] K. Jurczuk, M. Kretowski, J.-J. Bellanger, P.-A. Eliat, H. Saint-Jalmes, and J. B'ezyWendling, "Computational modeling of MR flow imaging by the lattice Boltzmann method and Bloch equation," Magnetic resonance imaging, vol. 31, no. 7, pp. 1163-1173, 2013.

[26] R. Marazzato and A. Sparavigna, "Fractional differential processing for medical imaging," Secondo Congresso Nazionale di Bioingegneria (Congresso GNB2010) ,Torino, 8-10 luglio, Italy, 2010

[27] Q. Yang, D. Chen, T. Zhao, and Y. Chen, "Fractional calculus in image processing: a review," Fractional Calculus and Applied Analysis, vol. 19, no. 5, pp. 1222-1249, 2016. 